\title{
1 Numerical prediction and experimental \\ 2 analysis of ends-together yarn splicing
}

3 Abstract

4 Pneumatic yarn splicing is a technical process for joining two yarn ends together. The

5 process involves injecting compressed air into a splicing chamber. Inlet pressure and

6 chambers slope determine the main parameters affecting this process. In this paper, large

7 eddy simulation (LES) of the flow field in four selected splicing chambers is carried out.

8 The chambers are used for splicing ends-together yarns. The results of these simulations are

9 analysed to investigate first the effects of the inlet pressure. Second, the effects of the

10 geometry of the chambers on the flow field inside the splicing chambers are determined.

11 These effects are studied and analysed to interpret the experimental results, which have

12 been obtained using the same splicing chambers. This provides further insight into the

13 parameters which are important to obtain good splicing characteristics. It is demonstrated

14 that the volume of the splicing chamber and the location of the air inlet channel play crucial

15 roles in the splicing of the end-together yarns. The root mean square (RMS) values of the

16 velocity magnitude inside a splicing chamber have predictive values for the retained splice

17 strength. The results provide solid evidence on the effectiveness of the CFD technology to

18 study pneumatic splicing and optimise the geometry of an ends-together splicing chamber. 


\section{Keywords}

2 Yarn splicing, ends-together, flow field, Large Eddy Simulation, splicing chamber

\section{Introduction}

4 Pneumatic splicing is a well-known technique in the textile industry. It is used for joining

5 two yarn ends together by injecting air with high speed into a splicing chamber. The

6 resultant joint needs to have the same properties as the original yarn, especially regarding

7 strength and appearance. The process of yarn splicing is done by consecutive steps. The

8 two yarn ends are placed together into the splicing chamber. The yarn can be introduced in

9 the splicing chamber from opposing ends or from the same side. The latter is called

10 superimposing or ends-together and this is studied in this work. The cover of the chamber

11 (the lid) is then closed and they are cut together with scissors. Next, a compressed and

12 turbulent air flow is injected into the chamber with high velocity. The aerodynamic forces

13 created by the flow field excite the yarn ends to move and, therefore, to splice them. These

14 forces first untwist the yarn ends and then splice them. This process affects both the quality

15 of the yarn being spliced and the productivity of the machine in which it is used because it

16 affects the subsequent process.

17 Yarn air splicing is simple in principle, but it is complex in details. Moreover, 18 observing the splicing is difficult due to the short time of the process and the small 
1 geometry of the splicing chamber, even with current camera technology. To date,

2 simulating yarn splicing is difficult because of the strong interaction of the yarn filaments

3 with each other and with the turbulent air jet. Due to this complexity, most previous studies

4 have been focusing on external parameters which can be measured and controlled and are

5 based on experimental results. For example Webb et al. ${ }^{1}$ studied the influence of air

6 pressure and duration on the splice strength. However, Webb et al. ${ }^{2}$ verified that

7 Computational Fluid Dynamics (CFD) can be used to analyse the flow field inside an

8 empty splicing chamber (with the absence of yarns). They found good agreement between

9 the airflow simulation and the results of a visualisation technique. Zhou and Qin. ${ }^{3}$ carried

10 out numerical simulations of the flow field inside different splicing chamber geometries,

11 they confirmed the effectiveness of CFD in studying pneumatic splicing. The process of

12 yarn splicing is commonly divided into two stages for the opposing ends splicing type of

13 chamber: the untwisting stage and the mingling stage. The untwisting stage is important to

14 prepare the yarn ends to be spliced. It affects greatly the quality of the splicing. Xing and

15 Ye. ${ }^{4}$ used the k-epsilon model to simulate the flow field inside an untwisting pipe of a

16 splicing chamber. By analysing the flow field, they discussed the principal of yarn

17 untwisting and their conclusions confirmed the pneumatic splicer makers' suggestions

18 about the principal of yarn untwisting. Wu et al. ${ }^{5}$ investigated the structural parameters 
1 which affect the untwisting stage. They made comparisons between the flow field

2 simulation and experimental data. They determined the geometrical parameters that affect

3 and improve the performance of untwisting yarn ends. In comparison, simulating the yarn

4 ends inside the chamber during the splicing is complex and has not been published yet. Cai

5 and Oxenham. ${ }^{6}$ used an one-way coupling method to model fibre movements. The flow

6 field data was used to calculate the fibre displacements, but no feedback to the flow field

7 was taken into account. Few articles on yarn splicing are based on numerical optimisation.

8 Degong et al. ${ }^{7}$ studied the influence of the inlet pressure on the velocity and pressure inside

9 a splicing chamber. In this way they could determine the best inlet condition for particular

10 chamber geometry. Wang et al. ${ }^{8}$ concluded that a rounded shape of a splicing chamber is

11 better than a square or a hexagon.

12 Moreover, when investigating yarn splicing experimentally, CFD simulation can 13 provide additional insights based on the simulated flow field to interpret the experimental 14 results. However, there is no common way to discuss and study yarn splicing due to the 15 diverse geometry of splicing chambers, which are used by companies, and the variety of the 16 yarns to be spliced. This is the reason why in the previous studies a specific splicing 17 chamber with specific yarns has been studied and discussed. 
The geometry of the splicing chamber, the air jet pressure and velocity and the

2 duration of the air injection must be chosen carefully to result in yarn splicing with good

3 quality. The shape of the splicing chamber must be designed to drive the airflow creating

4 the flow pattern needed for the splicing goal (e.g. create vortices Wang et al. ${ }^{8}$ and $\mathrm{Wu}$ et

5 al. $^{9}$ ). The air jet pressure and velocity should be appropriate to create sufficient

6 aerodynamic forces, but when they become excessive the appearance and the quality of the

7 yarn will be influenced Webb et al. ${ }^{10}$. In addition to the air injection duration, these three

8 factors are the main parameters that influence the splicing quality.

The basis of this paper is an experimental study on ends-together yarn splicing

10 chambers, by De Meulemeester et al. ${ }^{11}$. As opposed to the typical untwisting stage in most

11 ends-opposed splicers, no untwisting stage is present in this study. In that work, it was

12 shown that chamber size is important to obtain good splices, as well as the symmetry of the

13 chamber and the cutting position (length along which the vortices can act). Inlet pressures

14 above a certain threshold did not influence the splices much. Our aim is to use CFD to

15 obtain insights into why these experimental conclusions are obtained. No previous studies

16 of CFD on ends-together splice chambers are available.

17 In this paper, large eddy simulation of the airflow in four selected ends-together 18 splicing chamber geometries is carried out with two inlet pressures. The simulations are 
1 carried out in empty chambers, without yarn ends being present in the chambers. The effect

2 of the air pressure and the effect of the geometry of the splicing chambers are investigated.

3 Furthermore, the airflow characteristics are used to interpret the experimental results

4 obtained with these four chambers. Our conclusions will allow the construction of

5 improved ends-together splice chambers.

\section{Modelling and simulation}

\section{Chamber geometries}

8 Figure 1 (top) shows the different geometries of the chambers and the corresponding air

9 volume within the chambers (bottom). The air volume consists of two parts: the air inlet

10 channel and the splicing part. The air inlet channel is the cylindrical part. The splicing part

11 consists of the union of the V shape with the half cylinder. Figure 2 shows $2 \mathrm{D}$ views of the

12 CFD geometry for one of the chambers with indications of the important dimensions. The

13 axis of the air inlet channel is parallel to the y axis in chambers 1,2 , and 3 , whereas it

14 makes a $10^{\circ}$ angle with the y axis or the xy-plane in chamber 4 . Table 1 represents the

15 indicated dimensions of the four chambers, all chambers have the same length and height

$16 \mathrm{X}=16 \mathrm{~mm}$ and $\mathrm{Y}=10 \mathrm{~mm} . \Phi_{1}$ represents the diameter of the inlet channel, the location of

17 the inlet channel is made to be in the middle of chambers 1 and 2 while it is shifted to the

18 left in chambers 3 and $4 ; y_{1}$ is the height of the splicing part; $z_{1}$ is the width of the base of 
1 the V shape and its angle is equal for the fours chambers; $\Phi_{2}$ is the diameter of the half

2 cylinder, alpha $(\alpha)$ is the angle between the y axis and the axis of the inlet channel.

3 (insertFigure1)

4 (insertFigure2)

5 Table 1. Dimensions of the different geometries ( $\mathrm{mm}$ except for the last column in degrees)

\begin{tabular}{lccccc}
\hline Geometrical characteristics & $\Phi_{1}$ & $\mathrm{y}_{1}$ & $\mathrm{z}_{1}$ & $\Phi_{2}$ & $\alpha^{\circ}$ \\
\hline Chamber 1 & 4.5 & 5.1 & 2.5 & 8 & 0 \\
Chamber 2 & 4.5 & 3.6 & 1.5 & 5.8 & 0 \\
Chamber 3 & 4.5 & 5.1 & 2.5 & 8 & 0 \\
Chamber 4 & 4 & 5.1 & 2.5 & 8 & 10 \\
\hline
\end{tabular}

It can be seen from Table 1 that Chambers 1, 2 and 3 have the same inlet channel

7 diameter, whereas it is smaller in chamber 4. The intersection between the $\mathrm{V}$ shape and the

8 half cylinder is determined by the location of the air inlet channel. In chambers 1,2 and 3

9 the diameter of the half cylinder and the height of the inlet channel are dependent on each

10 other as the height of the chambers is fixed to fit in an automated splice cover. This means

11 that an increase in height of the cylindrical inlet part indicates a decrease in cylinder

12 diameter of the splicing part, like in chamber 2. Chamber 4 is made so that the splicing

13 part's dimensions are as for chamber 3, with different inlet channel diameter and location.

14 The main geometrical differences between the four chambers are as follows: chamber 2 is

15 the smallest chamber, chamber 1 and 3 have a different air inlet channel location, while 
1 chamber 4 is asymmetrical. The yarn ends are superimposed into the chamber (ends-

2 together) when they are inserted for splicing. They are fixed at the side towards the V part

3 (left part of a chamber in Figure 1) and they are free at the other side (right side).

\section{Simulation model}

5 The air flow which is used for yarn splicing is highly turbulent (the Reynolds

6 number is in the order of $10^{5}$ based on the vent hole width), Therefore, a turbulence model

7 must be used to handle the effects of turbulence in the flow field. In this work Large Eddy

8 Simulation (LES) has been used as turbulence model. In Fröhlich and Terzi. ${ }^{12}$ LES is

9 discussed mathematically and physically in detail, starting from Navier-Stokes equations

10 (Ferziger and Perić. ${ }^{13}$ ) which are the partial differential equations that describe the

11 dynamics of fluids. The basic idea of LES is that the large scales are calculated explicitly

12 and the small scales are modelled. Separating the scales is done by filtering the equations of

13 Navier-stokes. The filter is based on the mesh size which means that the simulation with

14 LES is sensitive to the mesh. The subgrid-scale Reynolds stress is the term that added to the

15 momentum equation in LES to close the equation (Fröhlich and Terzi. ${ }^{12}$ page 354). This

16 term contains the unresolved scales or the modelled ones. Although the cost of simulation

17 with LES is high, LES gives detailed information on the flow fluctuation and predicts the

18 jet properly. The numerical solutions were obtained using Ansys Fluent 14.5. 


\section{Grid generation and grid sensitivity}

2 The first step to carry out a numerical simulation of the flow field is to determine

3 the domain of the simulation and to divide it into cells (create the mesh or the grid). The

4 domain of calculation, in addition to a chamber, is extended sufficiently in the three

5 dimensions as shown in Figure 3. The mostly block structured meshes are created by

6 Gambit (Ansys Inc.). The white unmeshed regions in the grid in Figure 3 represent the

7 external wall of the chamber and the lid (the cover). The mesh is created to have the same

8 step size inside a chamber in the three directions $(\Delta x=\Delta y=\Delta z)$. Outside of a chamber, the

9 mesh is gradually stretched. A mesh sensitivity study has been performed to make sure that

10 the results of the simulations are independent of the mesh. The mesh sensitivity is carried

11 out with chamber 2 with inlet pressure 10 bar. Three mesh levels are tested. The total

12 number of cells in the three levels are 1.5, 2.5 and 4 million (grid 1, grid 2, and grid 3

13 respectively). To test the mesh sensitivity, the average velocities in yz planes are plotted in

14 Figure 4 where $\mathrm{x}$ ranges from $0 \mathrm{~mm}$ to $16 \mathrm{~mm}$ with step size equal to $1 \mathrm{~mm}$. This means for

15 each yz plane which corresponds to $\mathrm{x}$ value $(0: 1: 16 \mathrm{~mm})$ the average velocity is compared

16 in the different meshes. From Figure 4 it can be seen that the differences between grid 2

17 compared to grid 1 are bigger than grid 3 compared to grid 2. Although the errors between

18 grid 3 and grid 2 are small, the characteristics of grid 3 are chosen to carry out the 
1 simulations. Adopting a finer grid than gird 3 would increase highly the cost of the

2 simulations. Moreover, it is difficult to obtain an LES which is completely independent of

3 the mesh. Therefore, the characteristics of grid 3 are sufficient to have accurate results with

4 acceptable cost. The same mesh characteristics of grid 3 are used to carry out the

5 simulations for the four chambers. .

6 (InsertFigure3)

7 (InsertFigure4)

8 Boundary conditions

9 Two conditions of the inlet pressure were tested: 10 bar and 15 bar (relative to the

10 atmospheric pressure). The outlet condition is set to outlet pressure and the pressure equals

11 to the atmospheric pressure. The initial conditions are: the velocities are zero everywhere,

12 the pressure equals to 10 bar or 15 bar in the inlet channel and atmospheric pressure

13 elsewhere. With a time step equal to $5.10^{-6}$ seconds, the total time of 0.075 seconds was

14 simulated to have a solution that is statistically steady.

\section{Results}

16 For each chamber, inlet pressure 10 bar and 15 bar have been simulated. It has been

17 observed that the conclusions regarding the effect of the inlet pressure are the same for all 
1 chambers. Therefore, chamber 1 is chosen to represent the effect of the inlet pressure. Inlet

2 pressure 10 bar is chosen to investigate the effect of the splicing chamber geometry.

3 (InsertFigure5)

The results of the flow field will be presented in three planes, as shown in Figure 5.

5 First, the results in the chambers 1,2 and 3 will be presented and compared. Then the

6 results in the chamber 4 will be presented separately. Figure 6 shows the contours of the

7 mean velocity magnitude in plane 1 for the different inlet pressures for chamber 1 and for

8 the inlet pressure of 10 bar for chambers 2 and 3 . The airflow passes from the inlet channel

9 to the splicing chamber through the vent hole, an orifice determined by the intersection

10 between the two parts. It is known that when the flow passes through an orifice and if the

11 ratio of the total pressure upstream over the static pressure downstream is equal to or higher

12 than 1.89 , the flow is choked. This means that the flow passes through the throat section

13 with a velocity equal to the acoustic speed. This happens for all three chambers 1,2 and 3.

14 Behind the throat section, the airflow continues to expand inside the splicing chamber

15 forming an underexpanded jet which eventually runs into a normal shock. When the flow

16 reaches the wall of the lid, it divides in both directions. With the inlet pressure equal to 15

17 bar the flow expands to higher velocity than with the inlet pressure equal to 10 bar, but only

18 when the flow reaches the atmosphere. Therefore, we can conclude from Figure 6(a) and 
1 (b) that the inlet pressure does not affect the flow pattern inside the splicing chamber. Of

2 course it influences the mass flow rate ( $45 \%$ higher for the inlet pressure equal to 15 bar)

3 and the forces that can be executed on the yarn by the air jet, since the density of the fluid

4 will be higher with the higher inlet pressure.(InsertFigure6)

The geometrical effects on the flow field with inlet pressure 10 bar can be seen in

6 Figure 6(b) to (d). It can be seen in Figure 6(b) and (d) that the region of the jet flow for

7 both chambers shows not much differences. The air flow is totally expanded inside the

8 chamber 3 (see Figure 6(d)). It is seen that the values of the velocity are higher inside the

9 chamber than outside in Figure 6(b) and (d). The mean velocity magnitude in Figure 6(c)

10 for chamber 2 (the smallest chamber) shows that the flow did not expand sufficiently as in

11 the other chambers due to the smaller height of the splicing part. The maximum values of

12 the mean velocity are outside of chamber 2 , in the ambient air.

13 Above considerations show that one should not expect large changes in splicing

14 strength when increasing the pressure above 10 bar. By contrast, smaller chambers or

15 shorter chambers result in maximal velocity outside instead of inside the chamber,

16 suggesting that yarns will need to be cut longer to undergo the same effects. Apart from

17 this, a really small chamber like chamber 2 has less zones with high mean velocity. We can 
1 conclude that specific features of splice chambers are visible in the CFD analysis, but not

2 yet how splicing can happen at all. For this we consider flow directions.

Vortices are a major component of the turbulent flow. They have been identified to

4 play a crucial role in ends-opposed yarn splicing, and we assume that to be the case also in

5 the ends-together splice chambers. The vortices apparently make the yarn fibres intermingle

6 together. The geometry of a splicing chamber has a big impact on the formation of these

7 vortices. To demonstrate the created vortices in this type of chambers, chamber 3 is chosen.

8 Figure 7 shows velocity vectors in chamber 3 with inlet pressure equal to 10 bar, Figure

$9 \quad 7(\mathrm{a})$ and (c) show the mean velocity vectors in planes 2 and 3 respectively, Figure 7(b) and

10 (d) show instantaneous velocity vectors, at the last time step of the simulation, in the same

11 planes.

12 (InsertFigure7)

13 The velocity vectors represent the directions and the magnitude of the flow velocity.

14 It can be seen from Figure 7(a) that when the jet flow reaches the lid, it divides into two

15 directions forming two contra-rotating vortices. Figure 7(b) shows that the essential

16 changes in the directions and the values of the velocity vectors occur in the regions of

17 intersections between the vortices and the jet flow and in the centre of the vortices (right

18 and left of the jet flow). Figure 7(c) and (d) show that the flow consists of two vortices 
1 which interact in the middle of the chamber forming a violent region, especially in plane 3,

2 so to the right of the chamber. We believe that these rapid changes are a requirement for

3 splicing, allowing the mixing of yarn ends trapped in the left or right vortex.

4 The root mean square values of the velocity (RMS) are used to characterize the 5 amount of turbulence in the flow. It represents the deviations of the velocity from the mean

6 values. It provides an interesting insight regarding the splicing process in each chamber due 7 to turbulence effects, bringing forward those zones with the most violently changing 8 velocities. Mathematically, the root mean square of velocity is calculated as follows:

$9 R M S(V)=\sqrt{\text { mean }\left(V^{2}\right)-(\text { mean }(V))^{2}}$ where mean refers to time averaging at a certain

10 fixed point. In Fröhlich et al. ${ }^{14}$ the RMS of the velocity is used in many simulation results to 11 highlight the velocity fluctuations, or the velocity deviations from the mean values, and to 12 compare RMS values of LES and DNS (an example is shown in Fröhlich et al. ${ }^{14}$ page 417).

\section{3 (InsertFigure8)}

14 Figure 8 shows the contours of the root mean square (RMS) of the velocity 15 magnitude in plane 3 for the different chambers for an inlet pressure equal to 10 and 15 bar 16 for chamber 1 and 10 bar for the other chambers. For the three chambers, the highest RMS 17 values are located in the middle of each chamber, where the flow is changing most as has 
1 been shown already in Figure 7. The RMS values in chamber 1 show no significant 2 difference for the inlet pressure equal to 10 and 15 bar. The bigger geometries of chambers

31 and 3 allow higher fluctuations than the small geometry of chamber 2 . In chamber 3 , the

4 RMS values close to the wall of the chamber are somewhat higher than in chamber 1 . The

5 different pattern of the RMS contours between chambers 1 and 3 is due to the shifted

6 position of the vent hole, as is shown in Figure 6. We can assume that one yarn, or part of a

7 yarn, is trapped in one vortex, and the rest in the other, with the region with high

8 fluctuations allowing transfer of fibre strands from one vortex to the other. Transfer can

9 only happen after yarn has been sufficiently untwisted, after which retwisting (splicing) can

10 occur due to this chaotic nature of the air flow.

11 Chambers 1,2 and 3 are symmetrical geometries and the results presented above

12 show that these symmetrical geometries give almost symmetrical mean flow inside the

13 chambers, which agrees with our expectations. Conversely, chamber 4 is asymmetrical,

14 thus it is expected to give asymmetrical mean flow inside it. It is worth to investigate how

15 much the asymmetrical mean flow will affect the yarn splicing. Figure 9 shows the

16 contours of the mean velocity magnitude and the instantaneous velocity vectors with inlet

17 pressure 10 bar in the plane 3. Figure 9(a) shows that the flow inside chamber 4 is indeed

18 asymmetrical as there are high differences in the values of the mean velocity magnitude 
1 inside the chamber. In Figure 9(a) we see a region with low velocity and another with high

2 velocity. From Figure 9(b), it can be seen that, there is no clear formation of vortices inside

3 chamber 4 . Instead there is a rather chaotic flow. In comparison, the formation of vortices

4 in symmetrical chambers is clearly seen in Figure 7.

5 (InsertFigure9)

6 Experimental method

7 Experimental set up

8 The splice chambers used in the CFD study presented above were 3D printed and tested on

9 a splicing machine, as in De Meulemeester et al. ${ }^{11}$. The splicing machine can be used at 10 pressures from 5 to 15 bar with splice duration from $10 \mathrm{~ms}$ to $5000 \mathrm{~ms}$ and has a fixed 11 scissors position. The setup is given in Figure 10. Tests were done at 10 and 15 bar and a 12 duration of $1000 \mathrm{~ms}$. Above a splicing duration of $\sim 250$ milliseconds, splicing duration has 13 little influence on splice strength and was hence not varied. To show the influence of the jet 14 leaving the splice chamber, the cutting length was varied by cutting the yarns at the 15 standard position or at the chamber exit and then manually moving the scissors to cut the 16 yarns towards or away from the chamber exit in steps of $1.5 \mathrm{~mm}$.

17 (Insertfigure 10) 
As yarn we selected a common yarn used in tapestry splicing machines: a yarn

2 consisting out of 3 ring spun strands of about 250 tex each, with a resulting composed tex

3 number of 751.4 tex. The twist of the strands is $248 \mathrm{tpm}$, the twine is $147 \mathrm{tpm}$ and the

4 material is polyamide + wool. The strength of this yarn is $55.7 \mathrm{~N}$ with a standard deviation

5 of $5.0 \mathrm{~N}$, as tested on a Textechno Statimat M tensile tester. The yarn diameter is $2 \mathrm{~mm}$ with

6 porosity approximately equal to $40 \%$. Depending on the application, different evaluations

7 are possible of the resulting splices. In tapestry applications, the splice is normally not

8 visible and one is only interested in the resulting strength of the splice, which should be

9 sufficiently high to allow weaving. The value of interest is then the retained splice strength

10 RSS, defined as

$11 R S S=\frac{\text { Tensile strength post splice }}{\text { Mean tensile strength single not spliced yarn }}$ From a production point of view, the RSS should be as high as possible, as that

13 offers the best guarantee that the yarn will not break during the weaving process. In an 14 industrial tapestry setting a tensile strength test of $20 \mathrm{~N}$ after the splicing is common to test 15 if the splice is good or should be redone.

We start with comparing the 4 chambers at inlet pressure 10 bar and 15 bar for this

17 yarn. We also evaluate the chambers at different cutting lengths of the yarn. We consider 
1 the default position of the scissors as $0 \mathrm{~mm}$ and investigate an additional splice length of $2+1.5 \mathrm{~mm}$ and $+3.0 \mathrm{~mm}$.

\section{Experimental results}

4 The resulting average splice strength of 20 samples as in De Meulemeester et al. ${ }^{11}$ is listed 5 in Tables 2 and 3.

6 Table 2. Splice strength in $\mathrm{N}$ at 10 bar

\begin{tabular}{lcccccc}
\hline Splice length & $0(\mathrm{~mm})$ & $\mathrm{CV} \%$ & $+1.5(\mathrm{~mm})$ & $\mathrm{CV} \%$ & $+3.0(\mathrm{~mm})$ & $\mathrm{CV} \%$ \\
\hline Chamber 1 & 18.7 & 52 & 25.9 & 35 & 26.2 & 24 \\
Chamber 2 & 13.5 & 46 & 20.0 & 20 & 19.3 & 24 \\
Chamber 3 & 29.5 & 15 & 25.3 & 22 & 25.6 & 24 \\
Chamber 4 & 0 & & 0 & & 0 & \\
\hline
\end{tabular}

7

8 Table 3. Splice strength in $\mathrm{N}$ at 15 bar

\begin{tabular}{lcccccc}
\hline Splice length & $0(\mathrm{~mm})$ & $\mathrm{CV} \%$ & $+1.5(\mathrm{~mm})$ & $\mathrm{CV} \%$ & $+3.0(\mathrm{~mm})$ & $\mathrm{CV} \%$ \\
\hline Chamber 1 & 18.4 & 55 & 29.9 & 21 & 30.1 & 21 \\
Chamber 2 & 24.3 & 23 & 22.1 & 23 & 24.4 & 16 \\
Chamber 3 & 33.1 & 11 & 30.4 & 17 & 27.3 & 22 \\
Chamber 4 & 0 & & 0 & & 0 & \\
\hline
\end{tabular}

9

10 In spite of a low splice strength for chamber 1 at the standard cutting position with

11 inlet pressure 15 bar, we observe the lowest strength for chamber 2. One possible reason for 
1 this may be due to the small size of splice chamber 2 relative to the yarn. The yarn has little

2 space to move and friction of the yarn with the walls of the splice chamber is consequently

3 a lot higher. The strength changes with changing the cutting position: with inlet pressure 10

4 bar it increases at $1.5 \mathrm{~mm}$ and then decreases at $3 \mathrm{~mm}$, while the contrary happens with 5 inlet pressure 15 bar.

For chamber 1 with the standard cutting position of the scissors we observe a

7 dramatically lower strength. This is due to the small length of yarn ends extending past the

8 splice hole. During splicing, the yarn ends twist around each other, shortening the yarn

9 length to the right of the splice hole, while the yarn length to the left remains the same. This

10 often leads to premature ejection of the splice through the yarn entry hole to the left during

11 the splicing process, ejecting the splice before it has reached proper strength. Except of this

12 lower strength value for chamber 1 at the standard cutting position we observe a higher

13 strength than for chamber 2 because the chamber is also larger and allows easier movement

14 of the yarn within the splice chamber during splicing. The strength increases by extending

15 the cutting position $1.5 \mathrm{~mm}$ and $3 \mathrm{~mm}$ behind the standard cutting position, with only small

16 increase from $1.5 \mathrm{~mm}$ to $3 \mathrm{~mm}$.

17 For chamber 3 we observed the highest strength at the standard cutting position.

18 The reason for this is the larger length of the splice chamber towards the right of the splice 
1 hole. This allows more yarn to be contained within the splice chamber during the splicing

2 process. Even when the length of yarn towards the right shortens due to the twisting of the

3 ends around each other, there is still sufficient length such that the yarn is not ejected

4 prematurely out through the yarn inlet hole but remains in the chamber until the splicing

5 process is complete. The splice strength decreases when the cutting position and thus the

6 splice length is increasing.

For chambers 2 and 3 we observed higher strength at inlet pressure 15 bar than at

8 inlet pressure 10 bar, while this is not observed for chamber 1 at the standard cutting length.

9 By extending the cutting position by $1.5 \mathrm{~mm}$ or $3 \mathrm{~mm}$ we observe higher strength at inlet 10 pressure 15 bar than inlet pressure 10 bar for all chambers. In De Meulemeester et al. ${ }^{11}$, it

11 was found that splice strength increases rapidly from zero after a certain minimum pressure,

12 but then often reaches a plateau after which the strength no longer increases. For chamber 1

13 and 3 the splice strength reached a maximum at 12 bar, while the splice strength in chamber

142 kept increasing all the way up to the maximum achievable pressure of 15 bar.

With chamber 4 no splices could be made. The idea of an asymmetric entrance hole 16 was to create a more powerful vortex, as rotation of fibre strands around each other is how 17 splices are formed. Although the separate yarns untangle correctly with chamber 4 , the 18 fibre strands of the two yarns intermingle too weakly to form good splices. 
From the experimental study one can conclude that the splice chamber needs to be

2 large enough to contain the yarn which may allow sufficient motion during the splicing

3 process and that a correct cutting length is important to achieve the maximum splice

4 strength possible for a chamber at a given entry pressure. Instead of changing cutting

5 length, one can also provide a sufficient amount of chamber to the right of the splice hole,

6 which leads here to the strongest splices. Finally, an asymmetric entrance hole as

7 considered here in chamber 4 is not a viable way to construct a splice chamber.

\section{Discussion}

10 There is no apparent discrepancy in the values of the velocity magnitude inside the

11 chambers, for the inlet pressures of 10 or 15 bar. An inlet pressure higher than 10 bar gives

12 a higher mass flow rate as the density is higher. Moreover, as it has been observed from the

13 values of the root mean square of the velocity magnitude, the two inlet pressures provide

14 the same RMS values, which means that the velocity fluctuations remain constant with both

15 inlet pressures. Therefore, the vortices are identical. By consequence, for this type of

16 chambers, when the inlet pressure is higher than $10 \mathrm{bar}$, the yarn ends will be subjected to

17 the same airflow pattern with higher aerodynamics forces. The experimental results confirm

18 these observations. It is noticed that the splicing strength increases with increasing the inlet 
1 pressure on the standard and extended cutting position. However, it is not always so that

2 higher forces lead to better splicing. There is limit or threshold for the inlet pressure after

3 which there is no increase in the splicing strength or there is even a decrease, as it is shown

4 in the experimental results for chamber 1 at the standard cutting position.

By contrast, the velocity outside of the chambers is higher for higher inlet pressure

6 and this has an effect on the splicing. In some cases, it is observed that the yarn ends are

7 blown out of a chamber, especially when the inlet pressure increases. This means that the

8 aerodynamic forces which act on the yarn ends in that direction are considerable. The

9 aerodynamic forces could not be calculated because the simulation was carried out in

10 empty chambers. We investigate instead the calculated mass flow rate which may account

11 for this phenomenon. It has to be calculated at the left side of the chamber where $\mathrm{x}=0$. For

12 chamber 1 , it is higher $45 \%$ with inlet pressure 15 bar than with 10 bar. Furthermore, with

13 inlet pressure 10 bar it is $10 \%$ higher for chamber 1 than for chamber 3 . However, by

14 extending the cutting position at the right side of the chamber, the outer flow gives better

15 splicing strength to some extent. For example, the splicing strength increases with

16 increasing the cutting positions in chamber 1 . The flow did not completely expand inside

17 the chamber. When it goes out of the chamber, it continues expanding to higher velocities.

18 By consequence, the splicing strength is improved. 
The expansion of the flow is constrained by the dimensions of the chamber. The

2 bigger geometry of chambers 1 and 3 allows the flow to reach higher velocities inside them

3 than in chamber 2. The CFD analysis shows for chamber 2 lower maximum speeds and

4 lower RMS between the two vortices, which apparently reduces the maximum splice

5 strength that can be obtained at a specific inlet pressure compared to chambers 1 and 3 .

6 Moreover in Webb and Waters ${ }^{15}$ the effect of the cross section (or volume) of the splicing

7 chamber was analysed by an experimental study. They changed the yarn count to study the

8 influence of the cross section of the splicing chamber. They found that increasing the cross

9 section of a chamber improved the splicing strength, but when the yarn count became too big,

10 increasing the cross section did not improve the splicing strength. Here, in this work, the yarn

11 or the yarn count is the same with all chambers. On the one hand, the effect of the cross section

12 of the chambers with the same inlet pressure can be seen by flow characteristics as mentioned

13 above. On the other hand, with the smallest cross section, chamber 2, the splicing strength

14 improves when increasing the inlet pressure or increasing the cutting position. The numerical

15 and the experimental observations of the smallest cross section effects render difficult to draw a

16 definitive conclusion about whether these effects are related to the geometry of the chamber or

17 related to the flow features. It can be said that with a chamber whose cross section is small, 
1 the volume of this chamber compared to the yarn volume and the flow characteristics 2 together result in good or bad splicing quality.

The cylindrical inlet part shifted towards the entrance in chamber 3 leads to a

4 shifted flow field. This results in better splicing because the length of the flow field region

5 that contributes in the splicing is longer and the length of the yarn ends in the splicing

6 region are longer. Moreover, this region can be increased by changing the cutting position

7 for chamber 3, but the splicing strength is lower in this case because the flow will dissipate

8 in the ambient after leaving the chamber and too much yarn is present that needs to be

9 untwisted. It can be seen that chamber 3 has the highest splicing strength compared to the

10 others chambers on the standard cutting position.

11 The asymmetrical geometry of chamber 4 does not create the essential equal 12 strength vortices and fluctuating mingling zone, which are the principal factors to give 13 splicing. In fact, the experimental results for this chamber show that there is no splicing 14 produced at all. The flow is able to untwist the yarn ends but not to retwist them in a usable 15 splice due to the absence of the vortices. Additionally, it has been seen that the flow is 16 asymmetric with big differences around the chamber centre. This means that the forces are 17 also different: there is a region with high forces and another with low forces. Increasing the 18 inlet pressure from 10 bar to 15 bar did not change the flow pattern and no vortices are 
1 formed for either of the pressures. It rather increases the differences around the centre of

2 the chamber. By consequence, no splicing can be produced in chamber 4.

\section{Conclusion}

4 In this work, large eddy simulations of the flow field in four splicing chambers have been

5 carried out. Based on the numerical and experimental results presented in this paper, it can

6 be concluded that a splicing chamber will give splicing if it creates the flow field pattern

7 which is needed for splicing purposes: two large counter-rotating vortices and unstable

8 velocity directions at the interface of these counter-rotating vortices. The yarn ends will be

9 subjected to the same flow field with higher aerodynamics forces if a splicing chamber

10 supplied by an inlet pressure higher than 10 bar.

11 The volume of the splicing chamber plays an essential role in allowing the flow to 12 reach sufficiently high values of velocity, resulting in the necessary aerodynamic forces

13 that can make the yarn ends intermingling together, but these forces may not guarantee

14 good splicing if the geometry of a splicing chamber does not have sufficient space to let the 15 yarns intermingle together.

16 The location of vent hole or air inlet channel is important to create sufficient length

17 of the splicing region, which gives better splicing properties. A splicing chamber which is 
1 used to splice superimposed yarns should not have excessive amounts of backwards flow

2 (flow towards the left, not in the direction of splicing) because on the one hand, it does not

3 contribute in the splicing of the yarn ends and on the other hand it causes the yarn ends to

4 be blown out of chamber prematurely if it creates high aerodynamic forces.

5 Increasing the length of the splicing inside the chamber by changing the position of

6 the air inlet chamber is better than increasing it by changing the cutting position. The values

7 of the root mean square of the velocities (RMS) represent a new flow factor that shows the

8 level of turbulence inside a splicing chamber. One can predict the effectiveness of a

9 splicing chamber by looking to the RMS values inside it. Higher values of RMS are an

10 indication of more turbulent flow and this can predict good splicing.

\section{Acknowledgements}

12 This work was supported by Aleppo University of Syria and Ghent University of Belgium.

13

\section{References}

15 1. Webb CJ, Waters GT, Thomas AJ, et al. The use of the Taguchi design of experiment 16 method in optimizing splicing conditions for a Nylon 66 yarn. J Text Inst 2007; 98(4): 327336. 
2. Webb CJ, Waters GT, Liu GP, et al. The use of visualisation and simulation techniques to model the splicing process. J Text Inst 2010; 101(10): 859-869.

3. Zhou J and Qin P. Air flow in a pneumatic splicer by CFD. Text Res J 2005; 75(2): 106-110.

4. Xing $\mathrm{X}$ and Ye G. Numerical simulation of yarn untwisting mechanism in pneumatic splicer process. APPL MECH MATER 2012; 229-231: 1721-1724.

5. Wu Z, Shi P, Chen S, et al. Study on effects of structural parameters on untwisting performance in pneumatic yarn splicing. Text Res J 2015; 85(17): 1776-1788.

6. Cai Y and Oxenham W. Computer modeling of fiber movements in high-speed airflow. RJTA 2005; 9(4).

7. Degong C, Dengchen L, Songmei L, et al. Research on the impact of vent pressure on the yarn

8. Wang JZ, Zhou GZ, Ll L, et al. Air dynamics characteristics analysis of air splicer. Key Eng splicing based on ANSYS. Key Eng Mater 2014; 584: 50-53. Mater 2013; 561: 483-489.

9. Wu Z, Shi P, Chen S, et al. Study on the effects of the characteristics of a vortex on splice strength in pneumatic yarn splicing. Text Res J 2015. Epub ahead of print. DOI: $10.1177 / 0040517515588273$. 
1 10. Webb CJ, Waters GT, Thomas AJ, et al. Optimising splicing parameters for splice aesthetics for a continuous filament synthetic yarn. J Text Inst 2009; 100(2): 141-151.

3 11. De Meulemeester S, Malengier B and Van Langenhove L. Experimental investigation and 4 optimization of ends-together pneumatic splice chambers. Text Res J 2015. accepted for 5 publication.

12. Fröhlich J and Terzi DV. Hybrid LES/RANS methods for the simulation of turbulent flows. Prog Aerosp Sci 2008; 44: 349-377.

13. Ferziger JH. and Perić M. Computaional methods for fluid dynamics. $3^{\text {rd }}$ ed. Berlin: Springer 2002, p.3-10.

14. Fröhlich J, Kuerten H, Geurts B J, et al. Direct and Large-Eddy Simulation IX. ERCOFTAC Series, 1382-4309; 20: Springer 2015.

15. Webb CJ and Waters GT. The influence of yarn count on the splicing of simple continuous filament synthetic yarns. Text Res J; 79(3): 195-204. 


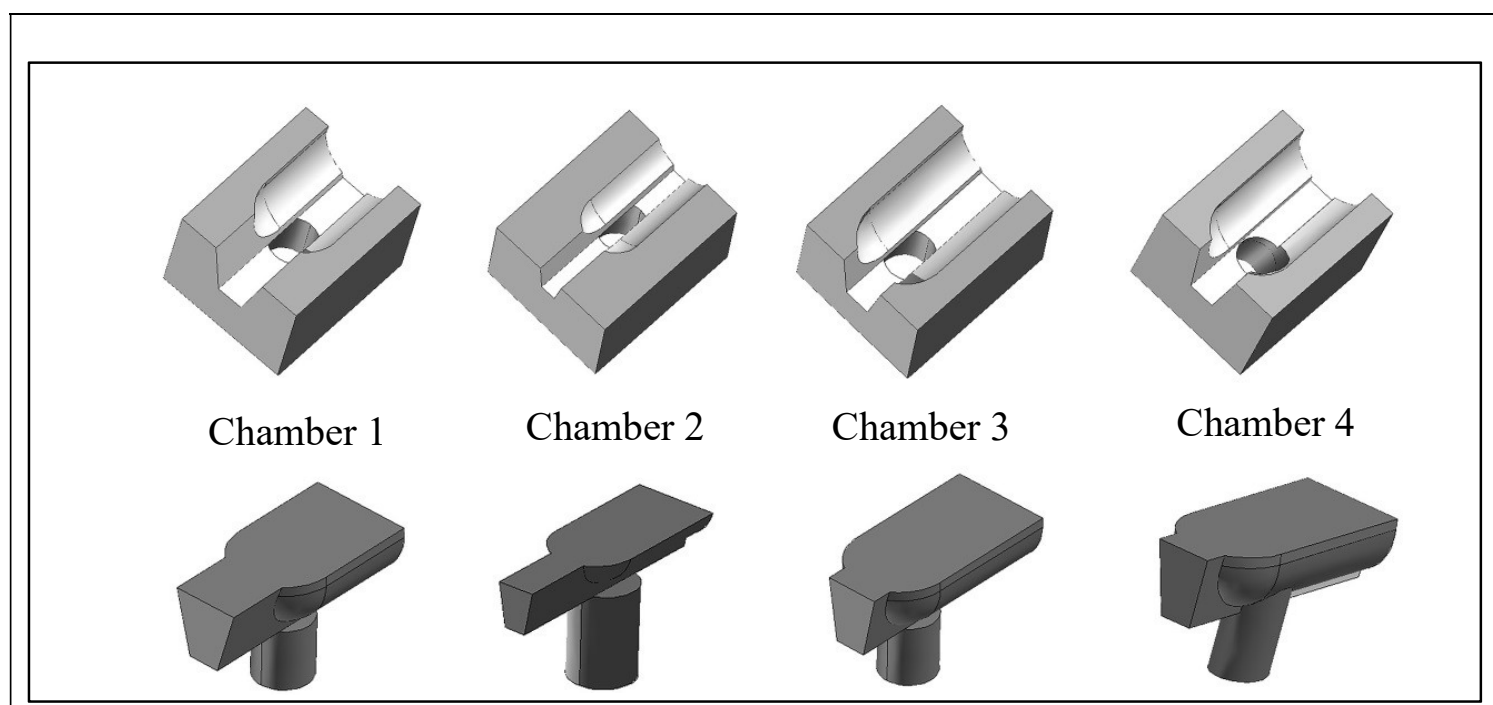

Figure 1 . The geometry of the chambers (top) and corresponding air volume inside the different chambers (bottom)

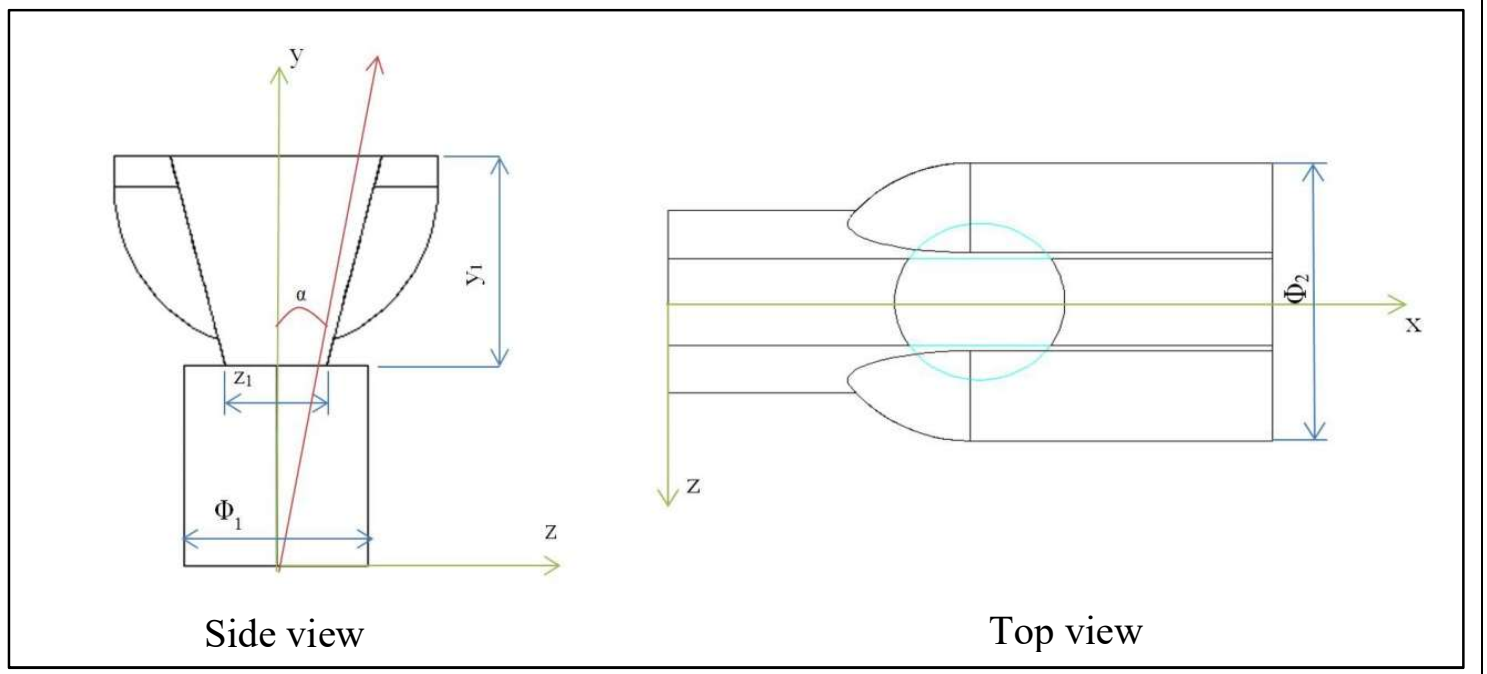

Figure 2. 2D views with geometrical characteristics of the chambers 


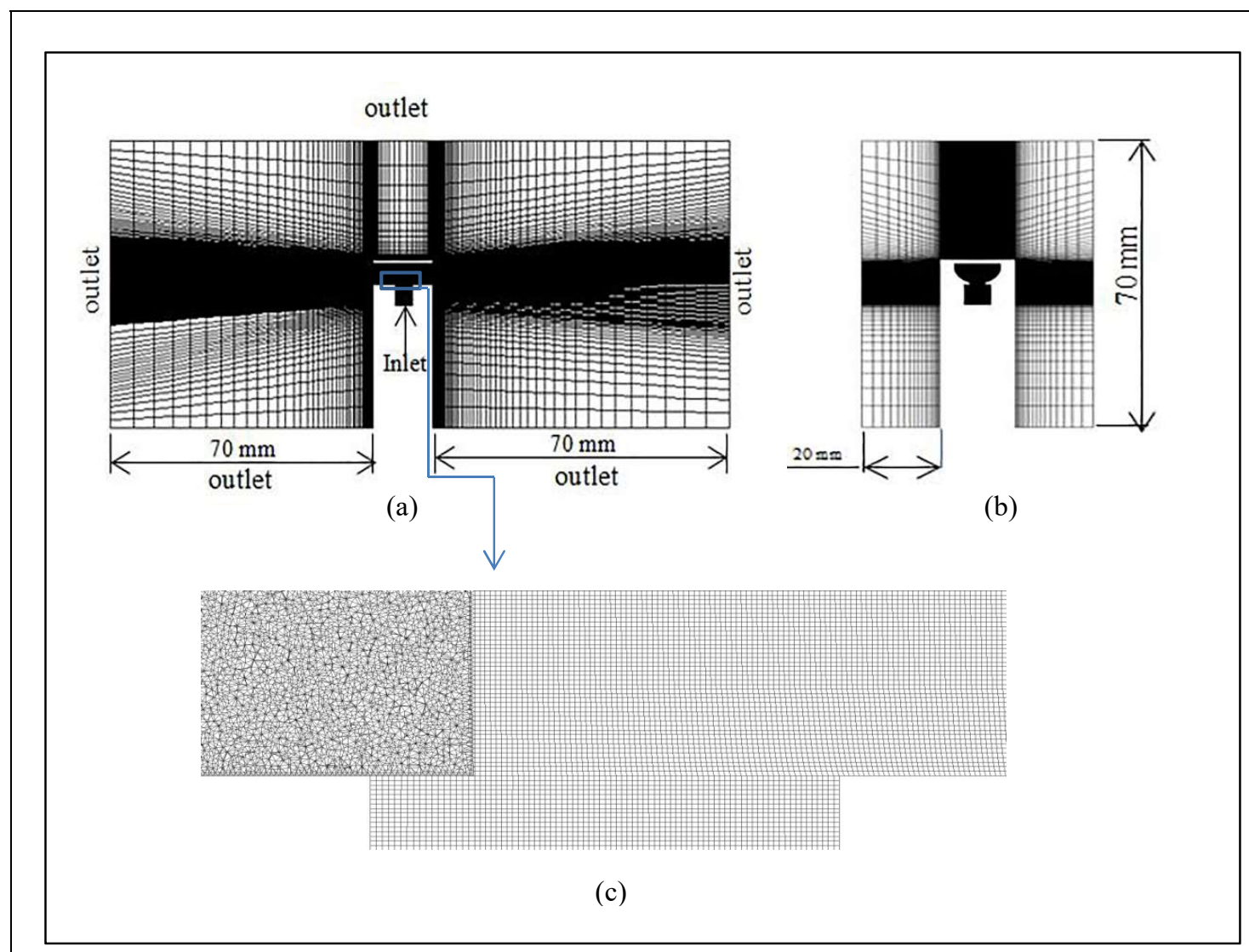

Figure 3. The flow grid: (a) the complete domain in xy plane; (b) the complete domain in yz plane ; (c) close view of the grid inside a chamber. 


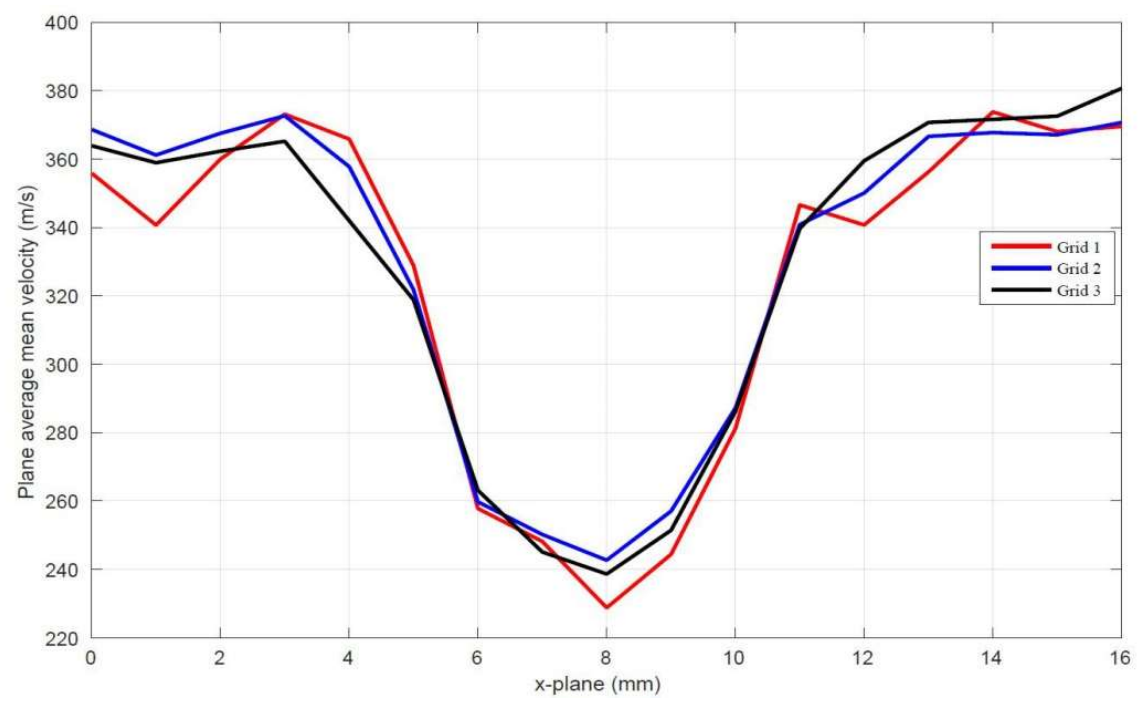

Figure 4. Grid sensitivity: (Vertical axis) the average mean velocity magnitude in the yz plane; (Horizontal axis) the $x$ coordinate of the yz plane : $x=0: 1: 16 \mathrm{~mm}$; (grid 1) the coarsest grid; (grid 3) the finest grid. 


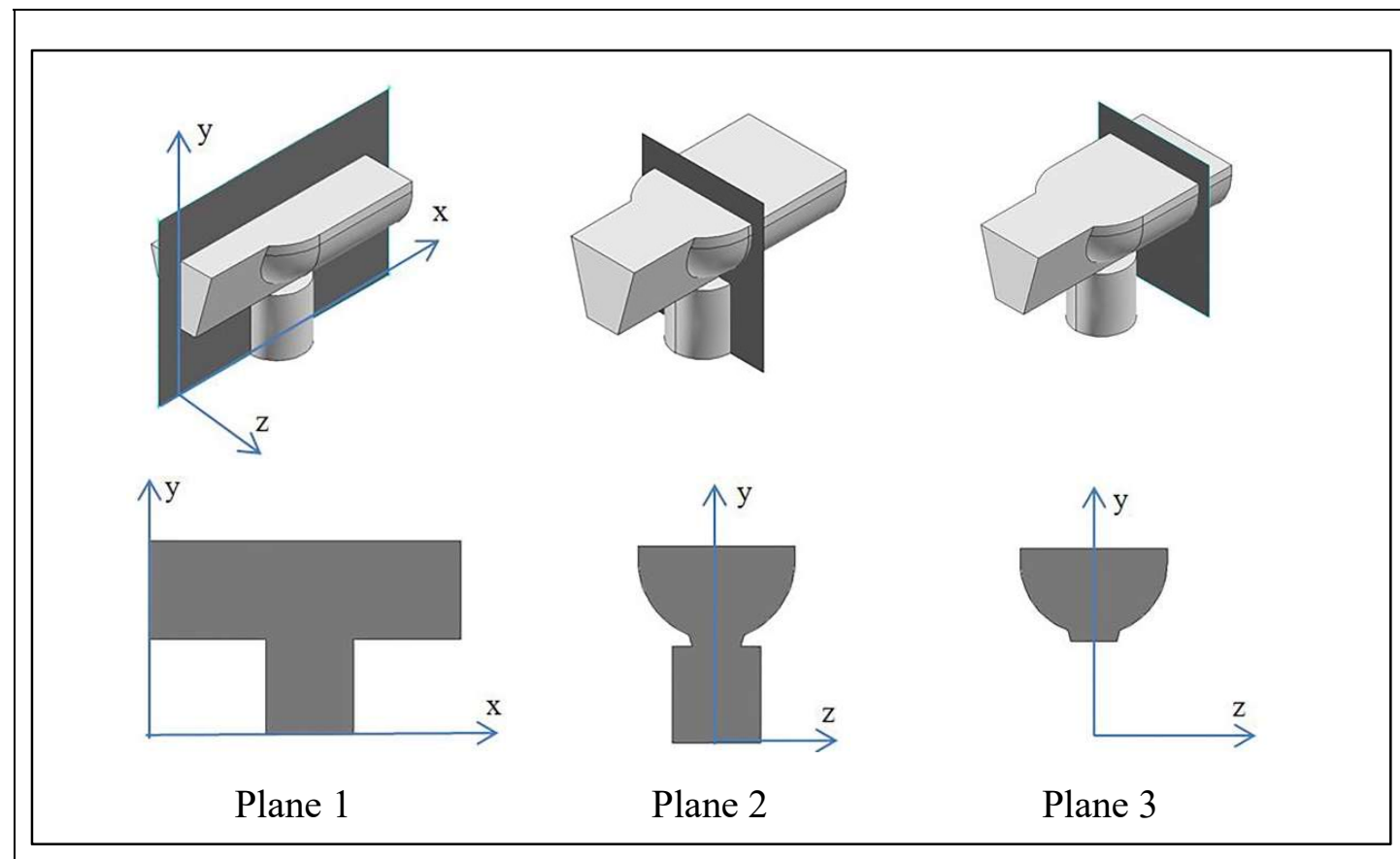

Figure 5. Definition of the 3 cut-planes: plane 1 corresponds to the xy-plane, which is the symmetry plane, planes 2 and 3 are parallel planes to the yz-plane, where $x$ corresponds to the location of the air inlet channel centre for plane 2 , and $x=13 \mathrm{~mm}$ for plane 3 , with the origin as indicated plane 1 . 


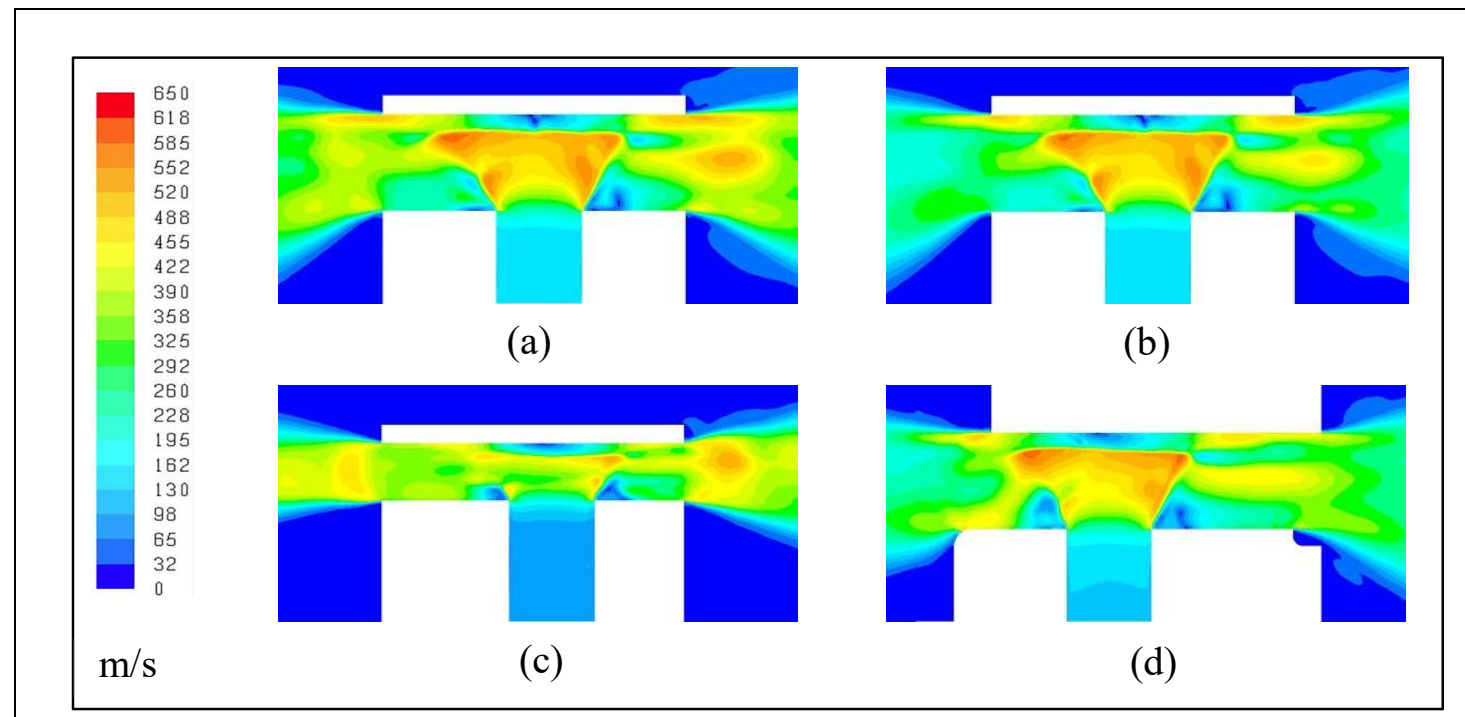

Figure 6. Contours of mean velocity magnitude in plane 1: (a) chamber 1, 15 bar; (b) chamber 1, 10 bar; (c) chamber 2, 10 bar; (d) chamber 3, 10 bar 


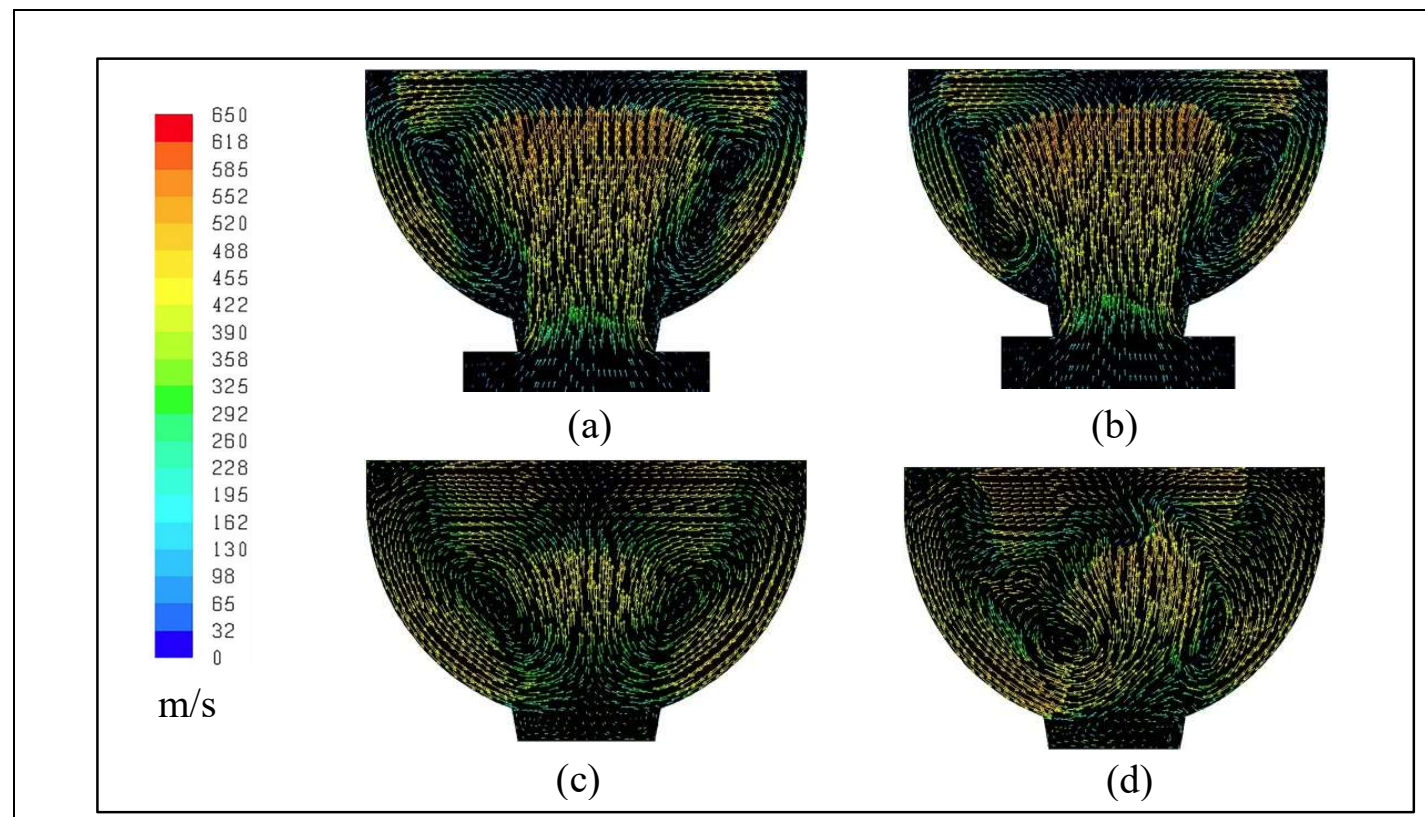

Figure 7. Illustration of velocity vectors in chamber 3 : (a) mean velocity vectors in plane 2 , (b) instantaneous velocity vectors in plane 2 , (c) mean velocity vectors in plane 3 , (d) instantaneous velocity vectors in plane 3 


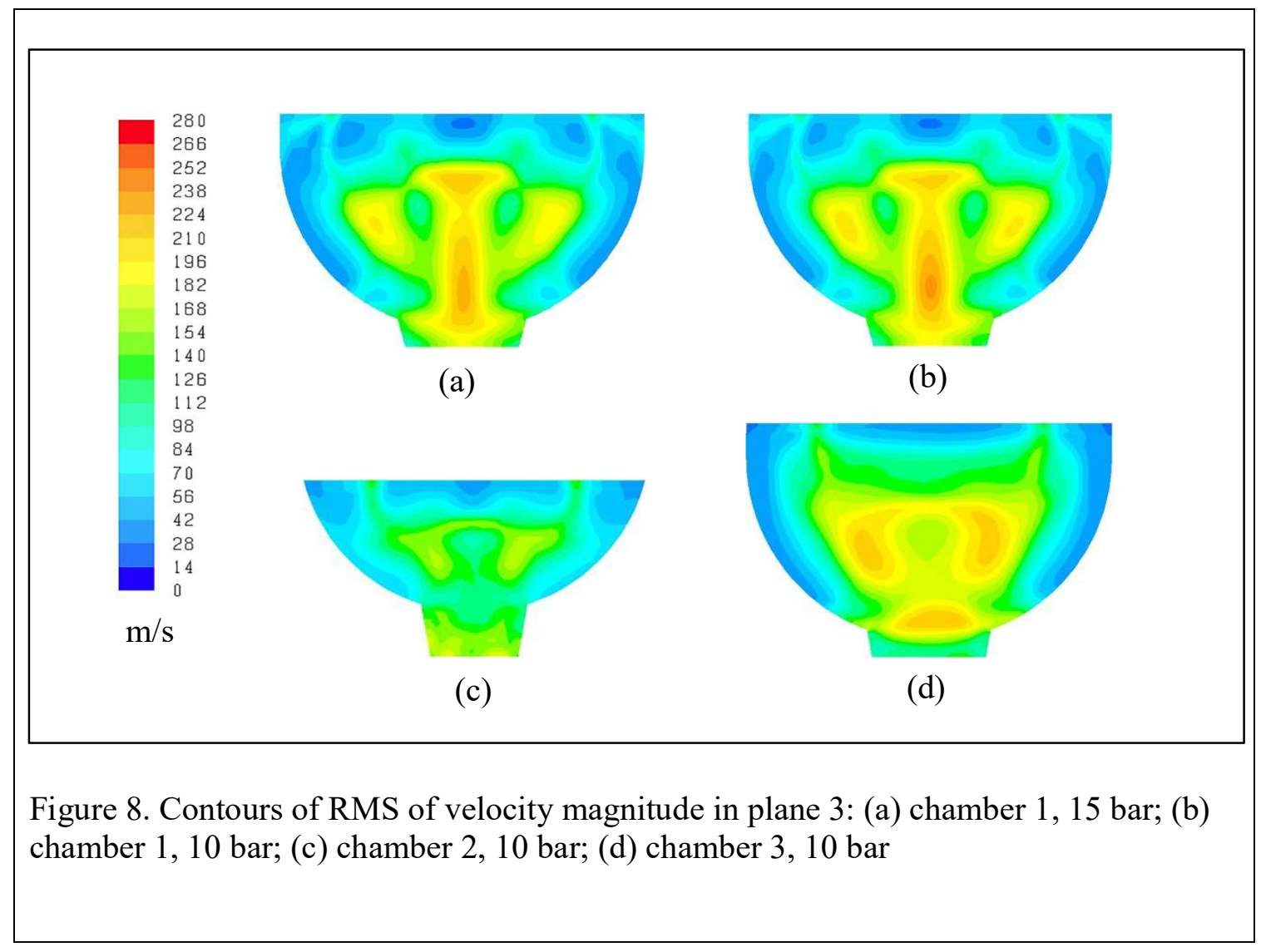




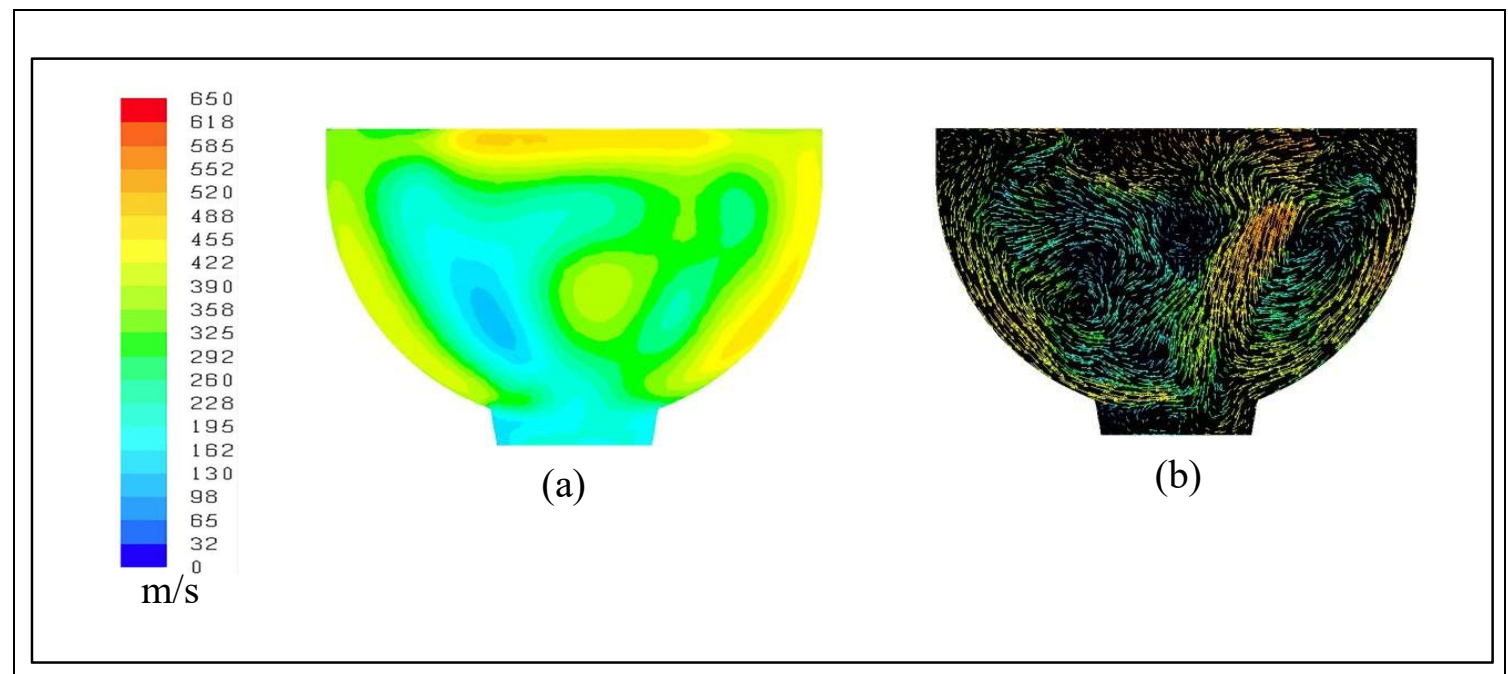

Figure 9. Contours of mean velocity magnitude and velocity vectors in chamber 4: (a) contours of mean velocity magnitude in plane 3 , (b) instantaneous velocity vectors in plane 3 


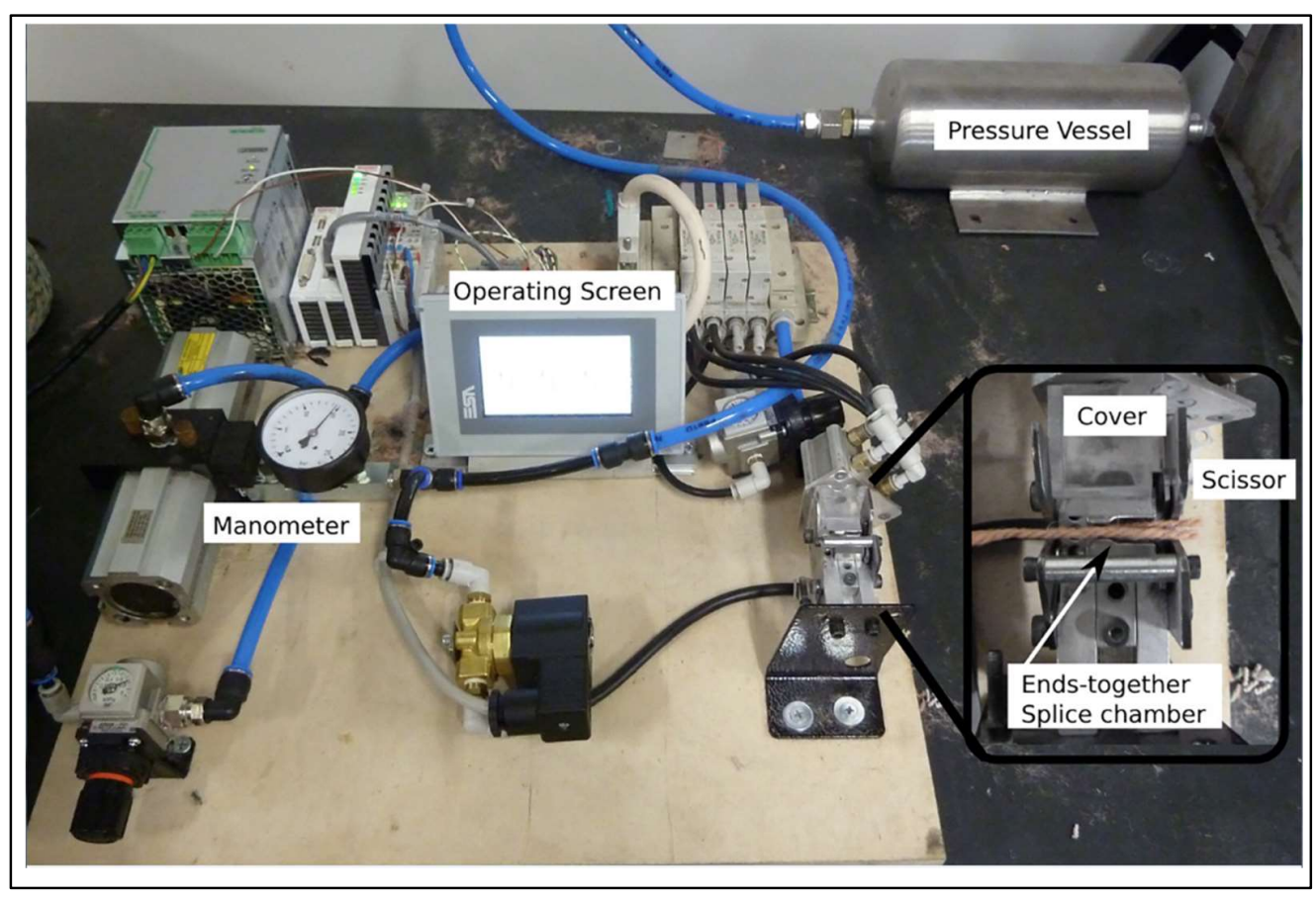

Figure 10. Experimental setup, with inset showing the ends-together splice chamber with cover and scissors 